\title{
Stroke-Like Presentation of Toxic Leukoencephalopathy as the Initial Manifestation of HIV Infection
}

\author{
Björn Reuter ${ }^{a} \quad$ Marc E. Wolf ${ }^{a} \quad$ Alex Förster $^{a} \quad$ Kira Kalvin $^{b}$ \\ Kristina Szabo $^{a}$ Hansjörg Bäzner ${ }^{a}$ Michael G. Hennerici ${ }^{a}$ \\ Departments of ${ }^{\mathrm{a}}$ Neurology and ${ }^{\mathrm{b}}$ Neuroradiology, Universitätsmedizin \\ Mannheim, University of Heidelberg, Mannheim, Germany
}

\section{Key Words}

Stroke mimic · Toxic leukoencephalopathy syndrome $\cdot$ HIV $\cdot$ Immunosuppression

\section{Abstract}

The toxic leukoencephalopathy syndrome is increasingly recognized, but rarely presents as an acute cerebrovascular syndrome. We report a 23-year-old man with sudden onset of a right hemispheric sensorimotor syndrome and final diagnosis of HIV-induced toxic leukoencephalopathy.

\section{Introduction}

Toxic leukoencephalopathy (TL) is characterized by MRI findings of subcortical edema without infarction, suspected to be caused by a partially reversible metabolic derangement and myelin as the most affected structure. This syndrome may develop in patients with exposure to environmental toxins (for instance carbon monoxide), drugs (heroin, cocaine, or methadone), and cancer chemotherapy (methotrexate, 5-FU, or capecitabine) [1].

\section{Case Presentation}

While studying in the university library, a male 23-year-old student developed a sudden and severe right hemispheric sensorimotor syndrome (National Institute of Health Stroke Scale (NIHSS) 9). The medical history was inconspicuous except for chronic diarrhoea during the last 8 months for unknown reason with subsequent weight loss of $13 \mathrm{~kg}$, and an Epstein-Barr virus infection in adolescence. No intake of medicines was reported. After clinical examination and inconspicuous 
cranial computed tomography, he was treated with IV rtPA $1 \mathrm{~h} 40$ min after symptom onset according to standard recommendations (ESO guidelines [2]). Under thrombolytic therapy, rapid improvement of the neurological deficits was observed (NIHSS 1 after thrombolysis, facial asymmetry). A 1.5 Tesla MRI after thrombolysis and $2 \mathrm{~h} 55 \mathrm{~min}$ after symptom onset revealed a diffusion-weighted imaging (DWI) hyperintense signal in the right and, to a lesser degree, in the left periventricular white matter with a corresponding apparent diffusion coefficient (ADC) reduction (fig. 1). Time-of-flight magnetic resonance angiography (TOF-MRA) showed bilateral vessel truncation of tertiary lateral hemispheric branches (fig. 2). Twenty-four hours after admission, the patient reported a slowly progressive hemihypesthesia of the left side over a period of $30 \mathrm{~min}$ and subsequent tetraparesis, dysarthria, and dysphagia were observed (NIHSS 12). The time from the second symptom onset to the maximum bihemispheric neurological deficit was $1 \mathrm{~h} 30 \mathrm{~min}$. A second MRI was performed $2 \mathrm{~h} 45 \mathrm{~min}$ after the second onset and demonstrated an almost symmetrical left hemispheric lesion with identical signal characteristics (fig. 1). Yet, TOF-MRA demonstrated reperfusion of the formerly truncated middle cerebral artery branches (fig. 2). The patient showed spontaneous and full recovery within $6 \mathrm{~h}$ and remained clinically stable for the following period of hospitalization (NIHSS 0). The stroke workup, including extra- and transcranial Doppler sonography, transesophageal echocardiography as well as $72 \mathrm{~h}$ cardiac and blood pressure monitoring, was unsuspicious. However, serologic studies revealed the diagnosis of a so far unknown HIV infection (viral load 720,000 copies/ml, CD4+ lymphocyte cell count $86 / \mu \mathrm{l}$ ). Serologic testing was negative for lues, hepatitis B and C, measles, mumps, varicellazoster virus (IgG positive, IgM borderline, complement-binding reaction and PCR negative), EpsteinBarr virus, Borrelia burgdorferi, Bartonella henselae, toxoplasmosis, and adenovirus. Cerebrospinal fluid (CSF) examination revealed no inflammatory state, with 1 leukocyte/ $\mu$, total protein amount $439.3 \mathrm{mg} / \mathrm{l}$, lactate $1.22 \mathrm{mmol} / \mathrm{l}$, and slightly reduced glucose $41 \mathrm{mg} / \mathrm{dl}$. Serum and CSF oligoclonal bands were equal and the Reiber diagram showed no signs of blood-brain barrier dysfunction or intrathecal immunoglobulin synthesis.

Further laboratory results were as follows: testing for cerebral vasculitis was negative; in detail, antinuclear antibodies, extractable nuclear antigen antibodies, anti-SS-A antibodies, anti-SS-B antibodies, anti-Sm antibodies, anti-Scl-70 antibodies, anti-Jo-1 antibodies, anti-U1-sn-RNP-Sm antibodies, rheumatoid factor, homocysteine, and complement system. The following screening parameters for hypercoagulopathy were within normal range: anticardiolipin antibodies, protein C and S activity, antithrombin III, factor VIIIc, and factor II PCR. D-dimer was elevated at $2.43 \mu \mathrm{g} / \mathrm{ml}$ (normal range $<0.5 \mu \mathrm{g} / \mathrm{ml}$ ) but fragmentocytes were not detectable, making a disseminated intravascular coagulation unlikely. Serum protein electrophoresis was normal, as was urinalysis for acute porphyria. An immunodeficiency-associated lymphoproliferative disorder and Whipple's disease were excluded by bone marrow analysis and endoscopy/coloscopy, respectively. However, advanced splenomegalia, HIV myelopathy, and HIV enteritis were diagnosed, thus indicating a longer lasting course of the disease.

A follow-up MRI examination prior to discharge home showed gradual regression of the subcortical edema, but a FLAIR image revealed hyperintense lesions (fig. 1). Under highly active antiretroviral therapy, our patient remained clinically stable, continued his studies and graduated from university.

\section{Discussion}

Acute TL may mimic cerebrovascular disease with initial involvement of the same structures and early demonstration of a DWI lesion, in this case involving the deep subcortical watershed [3]. However, with symmetrical lesion development and rapid clinical and MRI resolution it demonstrates the differentiating features between the two entities [4].

Today, a higher incidence of ischemic stroke in HIV-infected patients is a well-known complication $[5,6]$. Although stroke was our initial working hypothesis, we excluded possible underling pathophysiological mechanisms like opportunistic infections, cardioembolism, or coagulopathy. Cerebral vasculopathy in HIV usually presents with 
vasculitis, atherosclerotic stenosis, or aneurysmatic formation [6]. Serum and CSF laboratory findings did not support the diagnosis of cerebral vasculitis. Thus, we decided not to perform a catheter cerebral angiography, which would have been the gold standard for assessment of cerebral vasculitis. MR and ultrasound vascular imaging did not demonstrate aneurysms or stenoses of the brain-supplying arterial vessels, except for the transient and most likely vasospastic bihemispheric occlusion of tertiary middle cerebral artery branches. A previously published case report of an HIVpositive patient demonstrated improvement of multiple cerebrovascular stenoses with recurrent strokes suspected as HIV-associated arteriopathy [7]. Contrary to our case, vascular improvement took 5 months and was observed under successful highly active antiretroviral therapy.

Some further differential diagnoses should be discussed briefly. Transient vasoconstriction and subsequent ischemic stroke are typical findings in the reversible cerebral vasoconstriction syndrome [8]. However, in our case, the clinical presentation and recent medical history were negative for thunderclap headaches. The localization of DWI hyperintensities with an accompanying ADC reduction indicates an acute cytotoxic rather than a vasogenic edema, which is found in reversible posterior leukoencephalopathy syndromes (RPLS) [9]. Contrary to TL, focal vasoconstriction and vasodilatation have been demonstrated frequently in RPLS [10].

In the literature, TL is associated with drug abuse, environmental toxins, or cancer chemotherapy [1]. We did not determine any risk factor other than the recently diagnosed AIDS. As a limitation of our case, a drug screen at admission was not performed since the initial therapy was for ischemic stroke. On inquiry, the patient credibly disclaimed drug abuse. To our knowledge, temporary vasoconstriction of the supplying arterial vessels was not previously demonstrated in TL. A reason for our finding might be the repeated brain MRI, including TOF-MRA during the hyperacute phase of TL due to its sudden clinical onset, and immediate hospital admission. Altered cerebrovascular vasoreactivity in HIV-infected patients might be a result of viral vasculopathy [5, 11]. In 2008, Baehring and Fulbright [3] performed a PubMed-based review of the literature and collected 27 cases with a stroke-like presentation and the final diagnosis of TL shortly after administration of chemotherapeutic agents. Yet, an HIV-/AIDS-associated acute presentation of TL is uncommon. 

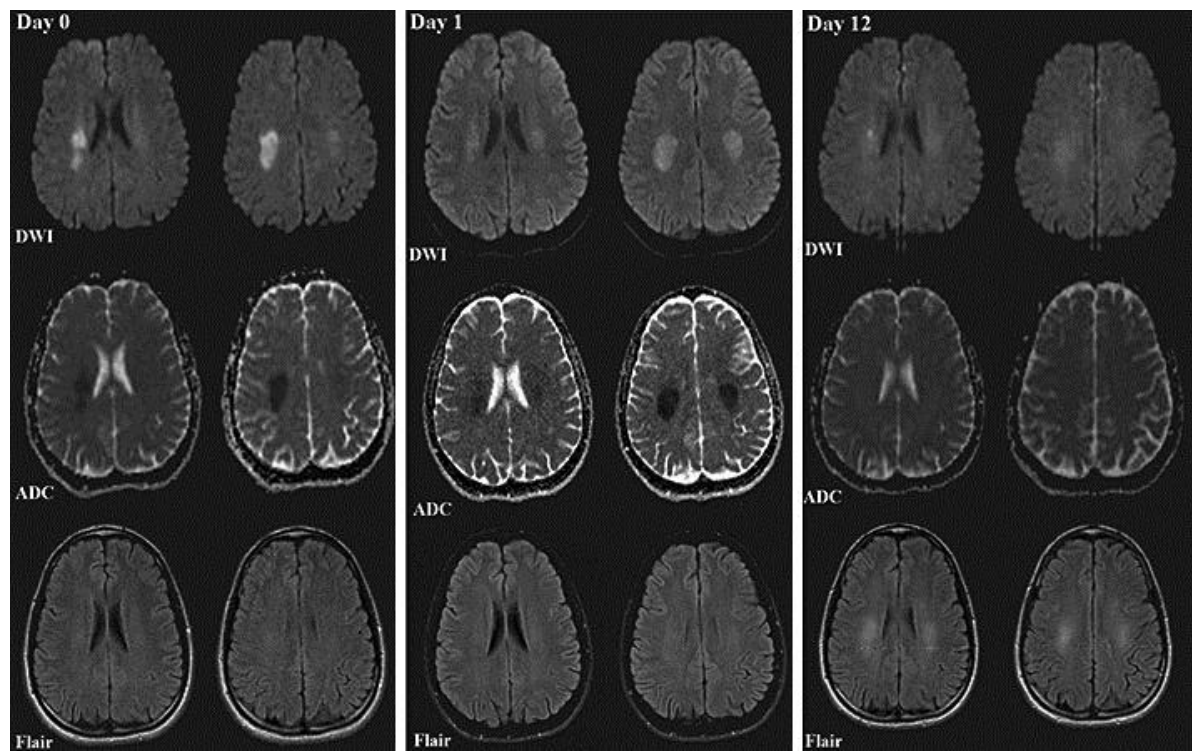

Fig. 1. Axial 1.5 Tesla magnetic resonance images (DWI, ADC and FLAIR) demonstrating bihemispheric white matter diffusion restriction in the corona radiata, finally with corresponding signal alteration in the FLAIR sequence.
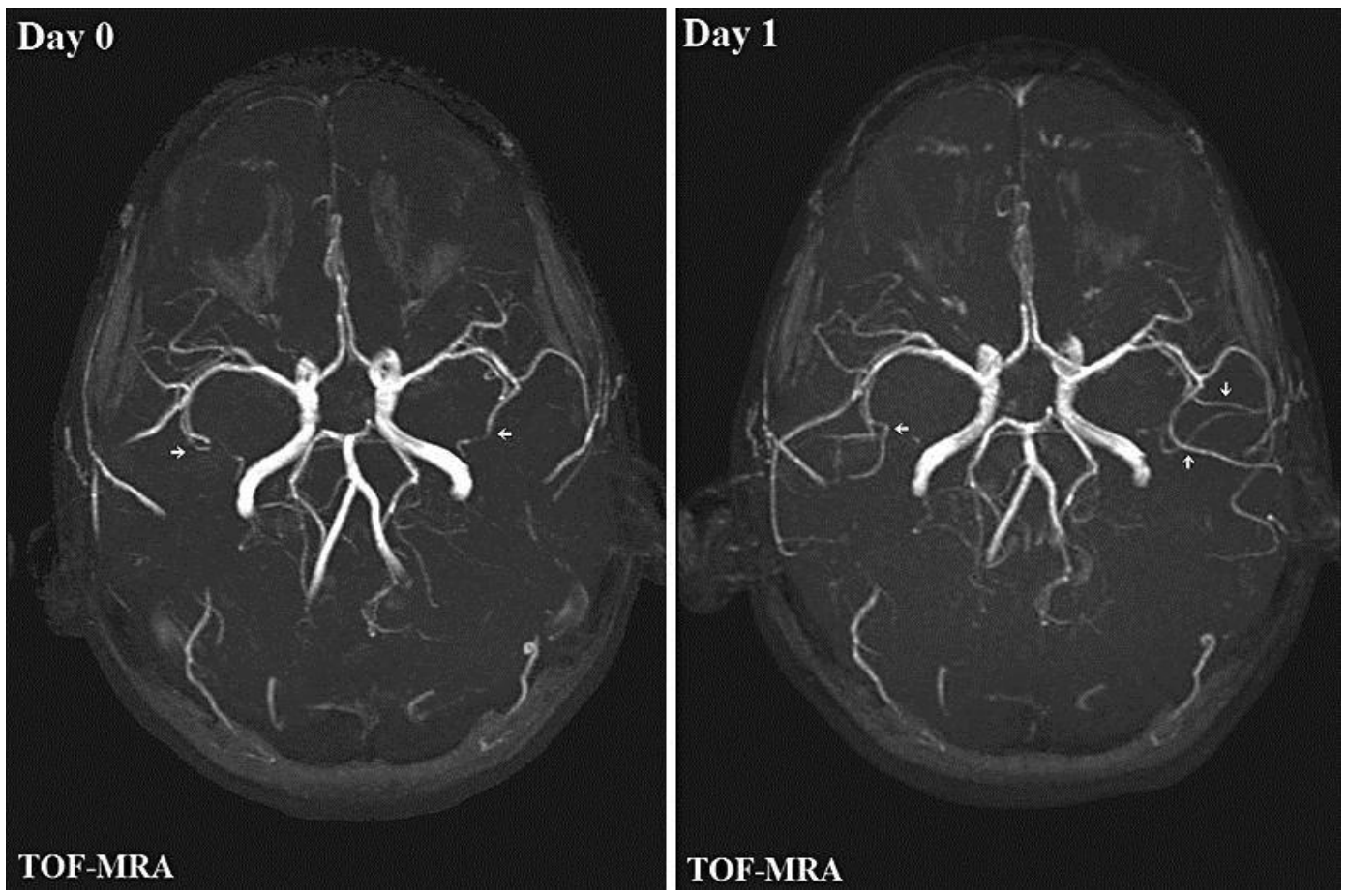

Fig. 2. 3D TOF-MRA presenting temporary symmetrical vessel occlusion of tertiary branches of both middle cerebral arteries. 


\section{References}

1 Filley CM, Kleinschmidt-DeMasters BK: Toxic leukoencephalopathy. N Engl J Med 2001;345:425-432.

$\checkmark 2$ European Stroke Organisation (ESO) Executive Committee; ESO Writing Committee: Guidelines for management of ischaemic stroke and transient ischaemic attack 2008. Cerebrovasc Dis 2008;25:457507.

3 Baehring JM, Fulbright RK: Delayed leukoencephalopathy with stroke-like presentation in chemotherapy recipients. J Neurol Neurosurg Psychiatry 2008;79:535-539.

4 McKinney AM, Kieffer SA, Paylor RT, SantaCruz KS, Kendi A, Lucato L: Acute toxic leukoencephalopathy: potential for reversibility clinically and on MRI with diffusion-weighted and FLAIR imaging. AJR Am J Roentgenol 2009;193:192-206.

5 Sen S, Rabinstein AA, Elkind MS, Powers WJ: Recent developments regarding human immunodeficiency virus infection and stroke. Cerebrovasc Dis 2012;33:209-218.

-6 Benjamin LA, Bryer A, Emsley HC, Khoo S, Solomon T, Connor MD: HIV infection and stroke: current perspectives and future directions. Lancet Neurol 2012;11:878-890.

7 Bhagavati S, Choi J: Rapidly progressive cerebrovascular stenosis and recurrent strokes followed by improvement in HIV vasculopathy. Cerebrovasc Dis 2008;26:449-452.

8 Ducros A: Reversible cerebral vasoconstriction syndrome. Lancet Neurol 2012;11:906-917.

9 Liman TG, Bohner G, Heuschmann PU, Endres M, Siebert E: The clinical and radiological spectrum of posterior reversible encephalopathy syndrome: the retrospective Berlin PRES study. J Neurol 2012;259:155-164.

10 Bartynski WS: Posterior reversible encephalopathy syndrome, part 1: fundamental imaging and clinical features. AJNR Am J Neuroradiol 2008;29:1036-1042.

11 Nagel MA, Mahalingam R, Cohrs RJ, Gilden D: Virus vasculopathy and stroke: an under-recognized cause and treatment target. Infect Disord Drug Targets 2010;10:105-111. 